\section{P367 HORMONAL CONTRACEPTION AND RISK OF STIS AND BACTERIAL VAGINOSIS IN SOUTH AFRICAN ADOLESCENTS: A RANDOMIZED TRIAL}

${ }^{1}$ Christina Balle*, ${ }^{2}$ Katie Lennard, ${ }^{3}$ Iyaloo Konstantinus, ${ }^{1}$ Shameem Jaumdally, ${ }^{1}$ Rachel Esra, ${ }^{1}$ Shaun Barnabas, ${ }^{4}$ Anna-Ursula Happel, ${ }^{1}$ Hoyam Gamieldien, ${ }^{5}$ Katherine Gill, ${ }^{6}$ Tanya Pidwell, ${ }^{7}$ Ulas Karaoz, ${ }^{8}$ Eoin Brodie, ${ }^{9}$ Venessa Maseko, ${ }^{10}$ Landon Myer, ${ }^{6}$ LindaGail Bekker, 'Jo-Ann Passmore, 'Heather Jaspan. ' University of Cape Town, Pathology, Observatory, South Africa; ${ }^{2}$ University of Cape Town, Cape Town, South Africa; ${ }^{3}$ University of Cape Town, Medical Virology, Observatory, South Africa; ${ }^{4}$ University of Cape Town, Medical Virology, Cape Town, South Africa; ${ }^{5}$ Desmond Tutu HIV Foundation, Desmond Tutu HIV Centre, Cape Town, South Africa; ${ }^{6}$ University of Cape Town, Desmond Tutu HIV Centre, Observatory, South Africa; 'Lawrence Berkeley National Laboratory, Berkeley, USA; ${ }^{8}$ University of California, Berkeley, USA; ${ }^{9}$ National Institute for Communicable Diseases, Sandringham, South Africa; ${ }^{10}$ University of Cape Town, School of Public Health, Observatory, South Africa

\subsection{6/sextrans-2019-sti.469}

Background Young women in sub-Saharan Africa are at high risk for sexually transmitted infections (STIs) and often rely on hormonal contraception (HC) to prevent unwanted pregnancies. Some observational data suggest that HC might affect STI risk. We examined the impact of three HC methods on the adolescent STI incidence and BV prevalence in a randomized trial.

Methods 130 adolescent females aged 15 to 19 from Cape Town were enrolled and randomized into three study arms: 1. injectable norethisterone enanthate (NET-EN), 2. combined oral contraceptives (COCs) or 3. combined contraceptive vaginal ring (CCVR) for 16 weeks. Participants then switched to a second HC for a final four months. Vaginal samples were collected at baseline, crossover and exit for STI (chlamydia, gonorrhoea, mycoplasma and trichomoniasis) and bacterial vaginosis (BV) testing by Nugent scoring.

Results At baseline, the BV and STI prevalence was $44 \%$ and $42 \%$, respectively. There were no significant differences in STI incidence between study arms at crossover, however in an according to protocol analyses, participants using COCs were significantly less likely to present with any STI than participants using either NET-EN (OR 0.22, 95\% CI 0.06-0.71, $\mathrm{p}=0.017$ ) or CCVR (OR 0.21, 95\% CI 0.05-0.69, $\mathrm{p}=0.015$ ). Specifically, participants on CCVR were more likely to be infected with N. gonorrhoea (OR 11.7, 95\% CI 2.0-224, $\mathrm{p}=0.025)$. These associations stayed significant after adjusting for sexual risk behaviour, including condom use. There was a decreased prevalence of BV in the COC arm and an increased BV prevalence in the NET-EN and CCVR arms at crossover, but these differences were not statistically significant.

Conclusion Use of COCs was associated with lower BV prevalence and STI incidence compared to NET-EN and CCVR use.

Disclosure No significant relationships.

\section{P368 LOW PREVALENCE OF VAGINAL DYSBIOSIS IN KENYAN ADOLESCENT GIRLS}

${ }^{1}$ Alison Roxby, ${ }^{2}$ Murugi Micheni, ${ }^{3}$ Stacy Selke, ${ }^{2}$ Lynda Oluoch, ${ }^{4}$ Tiffany Yuh, ${ }^{2}$ Bhavna Chohan, ${ }^{2}$ Catherine Kiptinness, ${ }^{5}$ Amalia Magaret, ${ }^{6}$ Kenneth Ngure, ${ }^{2}$ Nelly Mugo, ${ }^{4}$ Anna Wald*. ${ }^{1}$ University of Washington, Medicine and Global Health, Seattle, USA; ${ }^{2}$ Kenya Medical Research Institute, Center for Clinical Research, Nairobi, Kenya; ${ }^{3}$ University of Washington, Seattle, USA; ${ }^{4}$ University of Washington, Medicine, Seattle, USA; ${ }^{5}$ University of Washington, Laboratory Medicine, Seattle, USA; ${ }^{6}$ Jomo Kenyatta University of Agriculture and Technology, Community Health, Nairobi, Kenya

10.1136/sextrans-2019-sti.470
Background Vaginal dysbiosis is a risk factor for sexually transmitted infections (STI) and HIV in young women, and is highly prevalent in Africa. We sought to describe the microbiota of younger African women, before engaging in sexual behavior. Methods Adolescent girls aged 16-21 were recruited in Thika, Kenya. Eligible participants were HIV and HSV-2 seronegative, and reported sexual naiveté or one lifetime sexual partner. Nugent score was determined from vaginal Gram stains. Girls were tested for Neisseria gonorrhea (NG), Chlamydia trachomatis (CT), and Trichomonas vaginalis (TV). Chi-square testing was used to compare BV with other variables.

Results We enrolled 400 girls with a median age of 18.6 years (range 16-21). The majority (322 girls, 80.5\%) reported no history of sexual intercourse, while 78 (19.5\%) reported sex with 1 lifetime partner. Reported sexual partners were older, with median age of 22 years (IQR 19-25). At enrollment, 375 girls had vaginal Gram stains: 338 girls (90\%) had a normal Nugent score of $0-3$. BV (Nugent score $\geq 7$ ) was uncommon (21 girls, 5.6\%). Girls with BV were older (median age 19.3, IQR 17.9-20.3) than girls with Nugent score 0-3 (median age 18.6, IQR 17.6-19.3) and Nugent score 4-6 (median age 18.6, 17.9-19.8). Of 373 girls with STI testing, 49 girls were diagnosed with STIs and 7 girls had both STI and BV. BV was significantly associated with self-reported sexual intercourse: 52\% of girls with Nugent score $\geq 7$ reported prior sex, compared to $18 \%$ of those with Nugent score $0-3$ ( $\mathrm{p}=0.001$ ).

Conclusion Kenyan adolescent girls in a low-risk cohort have Lactobacillus-dominated vaginal microbiota, and only $5.6 \%$ of girls had evidence of BV. BV was found more often in girls who self-reported sexual intercourse. Interventions to prevent the onset of vaginal dysbiosis could be beneficial for African women.

Disclosure No significant relationships.

\section{P369 INCIDENCE OF VAGINAL INFECTIONS IN KENYAN WOMEN RANDOMIZED TO CONTINUOUS OR CYCLIC CONTRACEPTIVE RING (CVR)}

${ }^{1}$ Jeanne Marrazzo, ${ }^{2}$ Lei Wang, ${ }^{3}$ Nelly Mugo, ${ }^{4}$ Katherine Thomas, ${ }^{5}$ Kenneth Ngure, ${ }^{2}$ Kate Heller, ${ }^{2}$ Meighan Krows, ${ }^{5}$ Elizabeth Irungu. ' University of Alabama at Birmingham, Medicine/infectious Diseases, Birmingham, USA; ${ }^{2}$ University of Washington, Global Health, Seattle, USA; ${ }^{3}$ Kenya Medical Research Institute, Center for Clinical Research, Nairobi, Kenya; ${ }^{4}$ University of Washington, Seattle, USA; ${ }^{5}$ Jomo Kenyatta University of Agriculture and Technology, Community Health, Nairobi, Kenya

\subsection{6/sextrans-2019-sti.471}

Background Bacterial vaginosis (BV) is common in sub-Saharan African women, confers elevated risk for HIV acquisition, and is associated with menses. Antibiotic treatment provides symptomatic relief, but recurrence is common. Limited data support a favorable effect of a CVR containing estrogen and progesterone (NuvaRing) on vaginal bacteria, increasing quantities of favorable Lactobacillus species and reducing those of BV-associated bacteria (BVAB). We randomized women treated for BV in Thika, Kenya to continuous (menstrual suppression) vs cyclic (regular menses) use of NuvaRing, and assessed effects on BV.

Methods Women aged 18-40 years were enrolled and treated for BV defined by Amsel clinical criteria. One month later, they were randomized and evaluated monthly for 7 months. We used a logistic regression model with robust standard errors to compare BV incidence in each study arm at two 
months (immediate post-CVR) and 3-6 months (sustained post-CVR) relative to the 1 -month visit (pre-CVR).

Results Between April 2016 to November 2017, 151 women (median age 27 y) were enrolled and 122 (81.9\%) initiated CVR; 30 (24.6\%) were HIV-infected. Six women (4.9\%) had $\mathrm{BV}$ at the pre-CVR visit. Over a median duration of follow-up of 4.7 months, BV incidence/recurrence was $10.2 \%$ at the immediate post-CVR visit and $7.1 \%$ over the sustained postCVR visits. In a model combining CVR arms that adjusted for age and unprotected sex, we observed a non-significant increase in BV incidence/recurrence immediately post-CVR (adjusted OR $=2.5(0.9,7.2)$, after which BV returned to a level comparable to CVR insertion $(\mathrm{AOR}=1.2(0.8,1.9)$.

Conclusion Cumulative incidence of recurrent BV in the 6 months after CVR initiation is lower than historically reported rates in prospective studies, which are typically in $\geq 50 \%$ range. Concomitant incidence of vulvovaginal candidiasis, however, requires further study. The CVR should be considered for potential long-term optimization of the vaginal environment.

Disclosure No significant relationships.

\section{P370 PREVALENCE OF CHLAMYDIA, GONORRHOEA, M. GENITALIUM AND T. VAGINALIS IN THE GENERAL POPULATION OF SLOVENIA, 2016-2017}

${ }^{1}$ Irena Klavs*, ${ }^{2}$ Maja Milavec, ${ }^{2}$ Tanja Kustec, ${ }^{2}$ Marta Grgič Vitek, ${ }^{2}$ Darja Lavtar, ${ }^{2}$ Metka Zaletel, ${ }^{3}$ Andrej Golle, ${ }^{3}$ Darja Duh, ${ }^{3}$ Tjaša Žohar Čretnik. ${ }^{1}$ National Institute of Public Health, Communicable Diseases Centre, Ljubljana, Slovenia; ${ }^{2}$ National Institute of Public Health, Ljubljana, Slovenia; ${ }^{3}$ National Laboratory of Health Environment and Food, Maribor, Slovenia

\subsection{6/sextrans-2019-sti.472}

Background To inform sexually transmitted infections (STIs) prevention and control, objective of the second National Survey of Sexual Lifestyles, Attitudes and Health was to estimate the prevalence of Chlamydia trachomatis, Neisseria gonorrhoeae, Mycoplasma genitalium and Trichomonas vaginalis infections.

Methods A survey of the general population aged 18-49 was conducted in 2016-2017. We used stratified two-stage probability sampling from the Central Population Registry. Survey respondents were invited to contribute first void urine specimens for testing for C. trachomatis and unlinked anonymous testing for other STIs to obtain population prevalence estimates. Specimens were tested for C. trachomatis with specific real time PCR targeting both cryptic plasmid and bacterial chromosome. Positive results were confirmed by Sanger sequencing of the amplicon. Other STIs were detected by a commercially available multiplex PCR (FTD Urethritis plus, fast-track Diagnostics). To avoid false negative results, the human house keeping gene was amplified in all tested samples.

Results Urine specimens from 452 men and 635 women (56.4\% of all survey respondents) were tested for chlamydia. Overall weighted prevalence was $0.5 \% \quad(95 \%$ CI $0.1 \%$ to $1.4 \%$ ) in men and $1.7 \%$ (95\% CI $0.9 \%$ to $3.1 \%$ ) in women. Age-specific prevalence was the highest among 18-24 years old (men: $2.8 \%$; 95\%CI $0.9 \%$ to $8.5 \%$; women: $4.7 \%$; $95 \%$ CI $1.6 \%$ to $10.7 \%)$. Urine specimens from 430 men and 593 women $(53.0 \%$ of all survey respondents), were tested for other STIs. No infections with $N$. gonorrhoeae were found. Weighted prevalence estimate for $M$. genitalium was $0.5 \%$ (95\% CI $0.2 \%$ to $1.5 \%)$ in men and $0.3 \%$ (95\% CI $0.0 \%$ to $0.9 \%)$ in women. Parasite T. vaginalis was detected in one woman only. Corresponding weighted prevalence was $0.2 \%$ (95\% CI $0.0 \%-0.9 \%)$.

Conclusion The prevalence of C. trachomatis infection in the general population of Slovenians aged 18-24 was substantial. The other three STIs were relatively rare.

Disclosure No significant relationships.

\section{P371 EFFECT OF METRONIDAZOLE TREATMENT ON RECURRENT AND PERSISTENT BACTERIAL VAGINOSIS: A PILOT STUDY}

${ }^{1}$ Makella Coudray*, ${ }^{2}$ Daniel Ruiz-Perez, ${ }^{3}$ Brett Colbert, ${ }^{4}$ Karl Krupp, ${ }^{5}$ Hansi Kumari, ${ }^{2}$ Giri Narasimhan, ${ }^{5}$ Kalai Mathee, ${ }^{1}$ Purnima Madhivanan. ${ }^{1}$ Florida International University, Department of Epidemiology, Robert Stempel College of Public Health, Miami, USA; ${ }^{2}$ Florida International University, Bioinformatics Research Group, Miami, USA; ${ }^{3}$ Florida International University, Department of Biological Sciences, College of Arts and Sciences, Miami, USA; ${ }^{4}$ Florida International University, Department of Health Promotion and Disease Prevention, Robert Stempel College of Public Health, Miami, USA; ${ }^{5}$ Florida International University, Biomolecular Sciences Institute, Miami, USA

\subsection{6/sextrans-2019-sti.473}

Background This study aims to investigate the effect of metronidazole for the treatment of recurrent and persistent bacterial vaginosis (BV).

Methods Stored vaginal swabs of 80 African American (AA) women were randomly selected from a previously conducted clinical trial for this pilot study. Women with BV were treated with metronidazole. Vaginal smears were categorized by the Nugent score (NS) [0-3, normal; 4-6, intermediate state; 710, BV]. Women were classified as recurrent BV (RBV), persistent BV (PBV) or no BV based on three consecutive NS. RBV occurs when an episode of BV occurs after successful treatment of a prior episode. PBV occurs in instances when BV treatment fails to restore healthy Lactobacillus levels. All women were asymptomatic for BV at baseline and followed every two months for four months.

Results After four months, 22.5\% (CI: 13\%, 32\%) of women did not have BV, 7.5\% (CI: 2\%, 13\%) had RBV and 70\% had PBV (CI: 60\%, 80\%). 30\% of treated women did not have BV compared to $15 \%$ of untreated women $(p=0.18)$. BV recurred among $12.5 \%$ of treated women and $2.5 \%$ of untreated women $(\mathrm{p}=0.2)$. BV persisted among $57.5 \%$ of treated women and $82.5 \%$ of untreated women $(p=0.03)$. Women that were treated had 0.33 decreased odds $(95 \% \mathrm{CI}$ : $0.12,0.92, \mathrm{p} \leq 0.05)$ of having PBV as compared to untreated women. The mean age was 21.4 years (SD: 2.11 years). Prior antibiotic use among the sample was low (3.8\%), and $75 \%$ of women were not treated for BV during their lifetime. Among those who were previously treated for BV, $60 \%$ were treated more than five times. Douching was reported by $49 \%$ of the sample.

Conclusion These preliminary findings suggest, standard BV treatment may not be effective among women with RBV or adherence to treatment may be low among women with asymptomatic BV.

Disclosure No significant relationships. 